\title{
RHEED-AES Observation of Sb Desorption on a Single-Domain $\operatorname{Si}(001) 2 \times 1$ Surface $^{\dagger}$
}

\author{
Ki-Seon KIm, Yuji TaKaKuwa*, Tadashi AbuKawa and Shozo Kono \\ Research Institute for Scientific Measurements, Tohoku University \\ 2-1-1 Katahira, Aoba-ku, Sendai 980-77
}

(Received June 9, 1997; Accepted June 19, 1997)

\begin{abstract}
Sb desorption on a single-domain $\mathrm{Si}(001) 2 \times 1$ surface has been studied using grazing-incidence reflection high energy electron diffraction and Auger electron spectroscopy (RHEED-AES). From the time evolution of Sb coverage, $\theta_{\mathrm{sb}}$, during $\mathrm{Sb}$ desorption as monitored from Sb MNN Auger spectra, we have found that the Sb desorption kinetics are divided into two coverage regions; (I) $1 \mathrm{ML}>\theta_{\mathrm{sb}}>0.3 \mathrm{ML}$ and (II) $0.3 \mathrm{ML}>\theta_{\mathrm{sb}}>0 \mathrm{ML}$. It was also found that the Sb desorption is a first-order reaction in each coverage region with activation energies of (I) $3.61 \mathrm{eV}$ and (II) $3.63 \mathrm{eV}$ and pre-exponential factors of desorption coefficient of (I) $2.9 \times 10^{15} \mathrm{~s}^{-1}$ and (II) $5.0 \times 10^{15} \mathrm{~s}^{-1}$. This difference in pre-exponential factor is explained in term of the change in the morphology and structure of Sb layer as monitored by RHEED.
\end{abstract}

\section{Introduction}

$\mathrm{The} \mathrm{Sb} / \mathrm{Si}(001)$ system has attracted much attention in recent years because of its applications to surfactantmediated epitaxial growth ${ }^{1 \sim 3)}$ and $\delta$-doping, ${ }^{4,5}$. Since the applications are performed at high temperatures, $\mathrm{Sb}$ desorption essentially occurs, leading to the decrease in $\mathrm{Sb}$ surface coverage, $\theta_{\mathrm{sb}}$. Therefore, it is important to clarify the adsorption and desorption kinetics of $\mathrm{Sb}$ on $\mathrm{Si}(001)$ surface.

It was already reported that $\mathrm{Sb}$ grows in a StranskiKrastanov mode on a $\mathrm{Si}(001)$ surface below $\sim 150{ }^{\circ} \mathrm{C}$ and forms two-dimensional (2D) layer without islands above $\sim 150^{\circ} \mathrm{C}^{\text {) }}$. The saturation coverage of the $2 \mathrm{D} \mathrm{Sb}$ layer was observed to decrease with increase in temperature from 200 to $700{ }^{\circ} \mathrm{C}$ and the $\mathrm{Sb}$ saturation coverage is $\sim 1 \mathrm{ML}$ at $\sim 600{ }^{\circ} \mathrm{C}^{6,7)}$. The previous studies using low energy electron diffraction (LEED) ${ }^{8,9)}$ and scanning tunneling microscopy (STM) ${ }^{8,9)}$ have shown that the Sbsaturated $(\sim 1 \mathrm{ML}) \mathrm{Si}(001)$ surface has a $(1 \times 2)$ structure with defects such as voids and anti-phase domain boundaries. These reports also demonstrated that the $\mathrm{Sb}(1 \times 2)$ structure consists of Sb-dimers.

The desorption kinetics of $\mathrm{Sb}$ on $\mathrm{Si}(001)$ surface were first reported from thermal desorption spectra (TDS) by Metzger and Allen ${ }^{10)}$ to be a first order with an activation

\footnotetext{
†第16回表面科学講演大会（1996年11月 28 日〜 11月 29日） にて発表

*E-mail address: takakuwa@rism.tohoku.ac.jp
}

energy of $3.05 \mathrm{eV}$ for $\theta_{\mathrm{sb}}<\sim 0.5 \mathrm{ML}$. Barnett et al. ${ }^{\text {p }}$ later observed using temperature-programmed desorption (TPD) that $\mathrm{Sb}$ desorbs as Sb-monomers and $\mathrm{Sb}$ desorption occurs in a first-order reaction scheme for $\theta_{\mathrm{sb}}<$ $\sim 1 \mathrm{ML}$ at an activation energy of $2.33-2.40 \mathrm{eV}$. From medium-energy ion scattering (MEIS) measurements, Slijkerman et al. ${ }^{11)}$ reported that the Sb desorption reaction is a first-order for $\theta_{\mathrm{sb}}<\sim 0.75 \mathrm{ML}$ at an activation energy of $2.6 \mathrm{eV}$. Thus, all the previous reports agree that the desorption is the first order but with different activation energies. It is also noticeable that these reports gave no information on the surface structure and morphology of a $\mathrm{Sb}$-adsorbed $\mathrm{Si}(001)$ surface during $\mathrm{Sb}$ desorption. The morphology and the surface structure of Sb layers may be strongly related to the $\mathrm{Sb}$ desorption kinetics. For example, the $\mathrm{Sb}$ desorption kinetics were found to change in conjunction with a surface phase transition from $(2 \times 1)$ to $\alpha(2 \times 3)$ for the $\mathrm{Sb} / \mathrm{Si}(011)$ system $^{12,13)}$. It is, therefore, important to obtain in-situ the information on the surface structure as well as $\theta_{\mathrm{sb}}$ during $\mathrm{Sb}$ desorption on $\mathrm{Si}(001)$ at high temperatures.

In this study, we have employed a grazing-incidence reflection high energy electron diffraction and Auger electron spectroscopy (RHEED-AES) method to observe in-situ and simultaneously the surface structure and $\theta_{\mathrm{Sb}}$ during $\mathrm{Sb}$ desorption on $\mathrm{Si}(001)$. To simplify the analysis of surface structures during Sb desorption, a singledomain $(\mathrm{SD}) \mathrm{Si}(001) 2 \times 1$ surface was used as a substrate. An analysis of the time evolutions of the surface 
structure and $\theta_{\mathrm{sb}}$ is presented which leads to the reaction-order, the $\mathrm{Sb}$ desorption coefficient and the activation energy.

\section{Experimental}

The experiments were performed in an ultrahigh vacuum apparatus equipped with a grazing-incidence RHEED-AES system. Details of the apparatus are reported elsewhere ${ }^{14,15)}$. The base pressure was $\sim 2 \times 10^{-10}$ Torr and the pressure during the experiments was better than $\sim 5 \times 10^{-10}$ Torr. In the RHEED-AES method, we detected Auger electrons excited by a $10-\mathrm{keV}$ grazingincidence electron beam for RHEED observation. The incidence angle of the electron beam was $\sim 1.5^{\circ}$ from the $\mathrm{Si}(001)$ surface along the $\langle 100\rangle$ axis. For AES, we used a hemispherical electron energy analyzer with a mean radius of $132 \mathrm{~mm}$. The energy resolution of the analyzer was $\sim 1.0 \mathrm{eV}$. The input lens of the analyzer was oriented $\sim 80^{\circ}$ from the incident electron beam. Thus, AES spectra were taken at near surface normal during RHEED observation. RHEED patterns were recorded with a CCD video-camera system. RHEED intensity profiles were plotted from the recorded patterns using a commercial software.

The substrates were mirror-polished $\mathrm{Si}(001)$ wafers with a miscut angle of several tens seconds. The surface cleaning to obtain a SD $(2 \times 1)$ surface was carried out by flash heating at $\sim 1250{ }^{\circ} \mathrm{C}$ for $3 \mathrm{~s}$ followed by prolonged annealing at $\sim 1000{ }^{\circ} \mathrm{C}$ for $5 \mathrm{~min}^{16}$. The domain ratio of $(2 \times 1)$ to $(1 \times 2)$ was about 10 . Sb-saturated $(\sim 1 \mathrm{ML})$ $\mathrm{Si}(001)$ surfaces were made by depositing Sb of 99.9999 $\%$ purity onto a SD $\operatorname{Si}(001) 2 \times 1$ surface held at $\sim 617$ ${ }^{\circ} \mathrm{C}$. Then the substrate temperature was raised to a designated temperature for $\mathrm{Sb}$ desorption. The substrate temperature was monitored by an optical and an infrared pyrometer which was calibrated with an alumel/chromel thermocouple soldered with $\mathrm{Ag}$ to the surface of a test $\mathrm{Si}$ wafer for temperatures $300-800{ }^{\circ} \mathrm{C}$.

During Sb desorption, the intensity of Sb MNN Auger peak at $452.5 \mathrm{eV}$ was continuously monitored together with the RHEED intensities. The background intensity at $472.5 \mathrm{eV}$ was used to normalize the Sb MNN intensity for eliminating the effects of fluctuations in intensity and position of the primary electron beam.

\section{Results and Discussion}

Figure 1 shows an Sb MNN AES spectrum for the Sbsaturated $(\sim 1 \mathrm{ML}) \mathrm{Si}(001)$ surface together with electron spectrum for a clean substrate. The spectra were taken at $617^{\circ} \mathrm{C}$. Since the desorption coefficient of Sb was very small at $617^{\circ} \mathrm{C}$, the decrease in $\theta_{\mathrm{sb}}$ during this measurement was negligible. Because the signal-to-noise ratio is

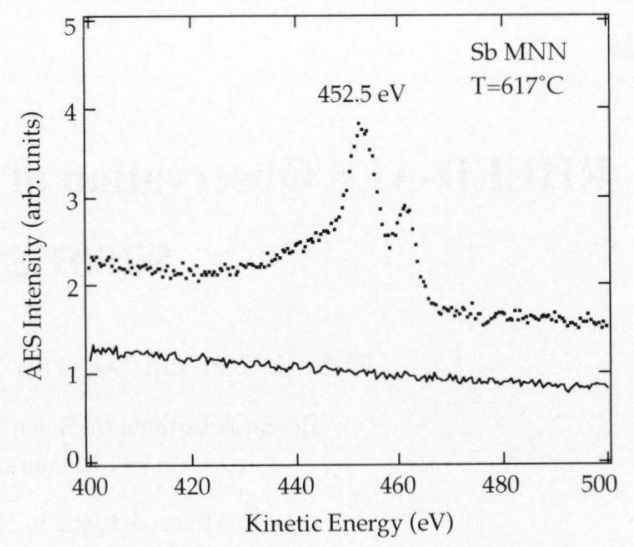

Fig. 1 Electron spectra for a clean single-domain $\mathrm{Si}(001) 2 \times 1$ surface (solid line) and the Sb-saturated $(\sim 1 \mathrm{ML})$ $\mathrm{Si}(001)$ surface (dotted line). The sample temperature during measurements was $617^{\circ} \mathrm{C}$. The incident angle and energy of the electron beam were $\sim 1.5^{\circ}$ and 10 $\mathrm{keV}$, respectively.

very high for $\mathrm{Sb}$ AES spectrum, no data-smoothing process was done. Two pronounced peaks appear due to Sb MNN Auger electrons. For a primary electron-beam with a sufficient current for RHEED, the counting rate of $\mathrm{Sb}$ MNN Auger intensity was the order of $\sim 10^{4}$ counts $/ \mathrm{s}$. As a result, it was possible to measure in-situ the time evolutions of $\theta_{\mathrm{Sb}}$ during $\mathrm{Sb}$ desorption. The precision in $\theta_{\mathrm{Sb}}$ measured was $\pm 0.05 \mathrm{ML}$.

Figure 2 (a) shows time evolutions of $\theta_{\mathrm{Sb}}$ in a logarithmic scale during $\mathrm{Sb}$ desorption at several temperatures. The $\theta_{\mathrm{sb}}$ decreases almost linearly at a larger rate for higher temperature. At $714{ }^{\circ} \mathrm{C}$, the desorption rate slightly increases at $\theta_{\mathrm{sb}} \sim 0.3 \mathrm{ML}$, showing an apparent break in the linear decrease of $\theta_{\mathrm{sb}}$. Such a break can be observed at almost the same $\theta_{\mathrm{Sb}}$ of $\sim 0.3 \mathrm{ML}$ for all the temperatures as indicated by a broken line in Fig. 2(a). Thus, the $\mathrm{Sb}$ desorption kinetics are divided into two $\theta_{\mathrm{sb}}$ regions; (I) $1 \mathrm{ML}>\theta_{\mathrm{sb}}>0.3 \mathrm{ML}$ and (II) $0.3 \mathrm{ML}>\theta_{\mathrm{sb}}>0 \mathrm{ML}$ as shown in Fig. 2(a). This change in the $\mathrm{Sb}$ desorption kinetics at $\theta_{\mathrm{Sb}} \sim 0.3 \mathrm{ML}$ has not been reported previous$1 y^{7,10,11)}$.

The straight lines in Fig. 2(a) are obtained by a leastsquares fit to each $\theta_{\mathrm{Sb}}$ curve. In both coverage regions, good linear correlations are observed, proving that the $\mathrm{Sb}$ desorption follows a first-order reaction scheme. An Arrhenius plot of the Sb desorption coefficient obtained by the above best-squares fits is shown in Fig. 2 (b). From the least-squares fit to the data points of Fig. 2 (b), the activation energy, $E_{\mathrm{a}}$, and pre-exponential factor, $k_{0}$, for $\mathrm{Sb}$ desorption are determined to be $3.61 \pm 0.05$ and $3.63 \pm$ $0.05 \mathrm{eV}$ and $2.9 \times 10^{15}$ and $5.0 \times 10^{15} \mathrm{~s}^{-1}$ in the regions I and II, respectively. It should be noted that there is a large 


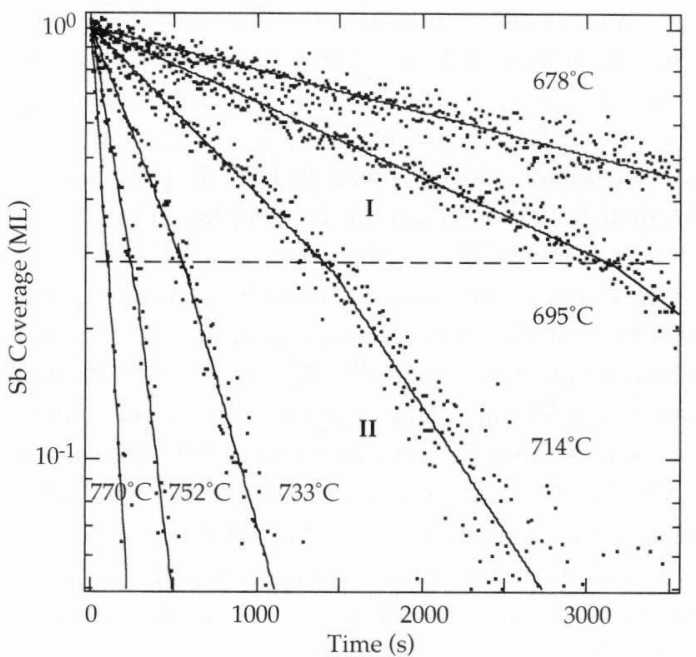

(a)

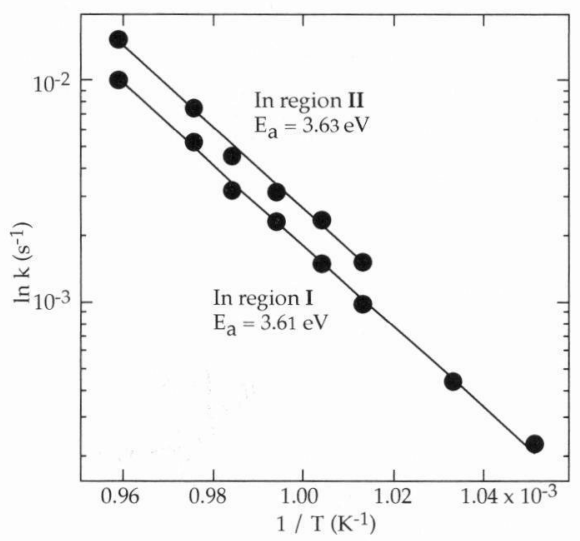

(b)

Fig. 2 (a) Time evolution of $\theta_{\mathrm{sb}}$ during Sb desorption plotted on a logarithmic scale for several temperatures. The straight lines are the least-squares fits to the data points. (b) Arrhenius plot of the rate of Sb desorption obtained from the slopes of the straight lines in (a).

difference in pre-exponential factor in comparison with the slight difference in activation energy. Consequently, the increase in $\mathrm{Sb}$ desorption coefficient at $\theta_{\mathrm{sb}}<0.3 \mathrm{ML}$ may be ascribed to the difference in the pre-exponential factor.

The present values of $E_{\mathrm{a}}$ are larger than those of 2.33$2.40 \mathrm{eV}$ by Barnett et al. ${ }^{7}, 3.05 \mathrm{eV}$ by Metzger and Allen $^{10)}$ and $2.6 \mathrm{eV}$ by Slijkerman et al ${ }^{11)}$. The present values for $k_{0}$ are also in disagreement with others; $5 \times 10^{9}$, $2.0 \times 10^{12}$ and $6 \times 10^{8}-3 \times 10^{11}$, for Refs. 7), 10) and $11)$, respectively. In their experiments, although $\mathrm{Sb}$ desorption was identified to be a first-order reaction, the $\mathrm{Sb}$ desorption kinetics were analyzed using a single first-

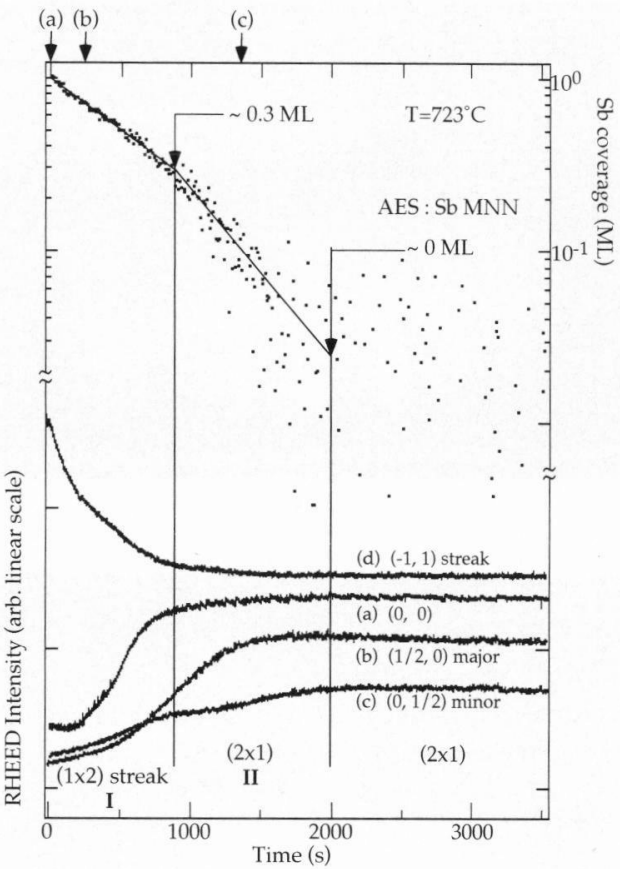

Fig. 3 Comparison of the time evolution of $\theta_{\mathrm{sb}}$ with those of four RHEED spot intensities during Sb desorption at 723 ${ }^{\circ} \mathrm{C}$. (a) $(0,0)$ specular spot for a measure of surface roughness; (b) $(1 / 2,0)$ spot for $(2 \times 1)$ periodicity; (c) $(0,1 / 2)$ spot for $(1 \times 2)$ periodicity; $(d)(-1,1)$ streak due to the $(2 \times 1),(1 \times 2)$, and $(1 \times 1)$ structures. The corresponding areas in RHEED patterns are marked with squares in Fig. 4.

order equation over an entire $\theta_{\mathrm{sb}}$ region. However, the $\mathrm{Sb}$ desorption kinetics change apparently at $\theta_{\mathrm{sb}} \sim 0.3 \mathrm{ML}$ as found presently. This may be the origin for the differences in $E_{\mathrm{a}}$ and $k_{0}$.

Figure 3 shows time evolutions of RHEED intensities during $\mathrm{Sb}$ desorption at $723^{\circ} \mathrm{C}$, compared with that of $\theta_{\mathrm{sb}}$. The RHEED intensities in Fig. 3 were measured for areas marked in photographs of RHEED patterns of Fig. 4. The four RHEED intensity profiles represent the time evolutions of (a), specular spot for the atomic smoothness of the surface; (b), $(1 / 2,0)$ spot for the area of domains with $(2 \times 1)$ periodicity; $(c),(0,1 / 2)$ spot for the area of domains with $(1 \times 2)$ periodicity; $(d),(-1,1)$ streak for the degree of order of $(2 \times 1),(1 \times 2)$ and $(1 \times 1)$ periodicities.

As $\theta_{\mathrm{sb}}$ decreases, the $(-1,1)$ streak intensity decreases drastically and the $(0,0)$ spot intensity increases rapidly and both the $(0,1 / 2),(1 / 2,0)$ spot intensities increase gradually. Around $\theta_{\mathrm{sb}} \sim 0.3 \mathrm{ML}$, the intensity changes of $(0,0),(-1,1)$ and $(0,1 / 2)$ become very slow having nearly a saturation behavior, whereas the $(1 / 2,0)$ spot 


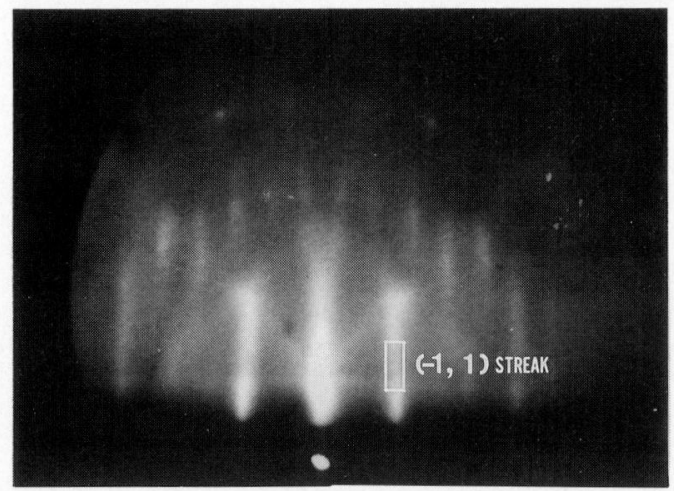

(a)

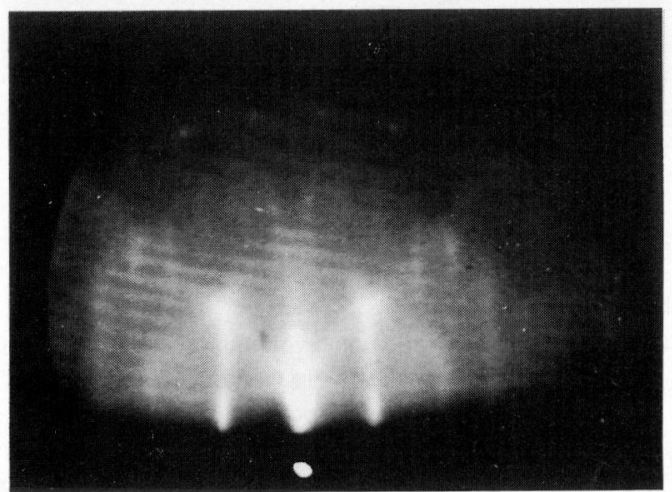

(b)

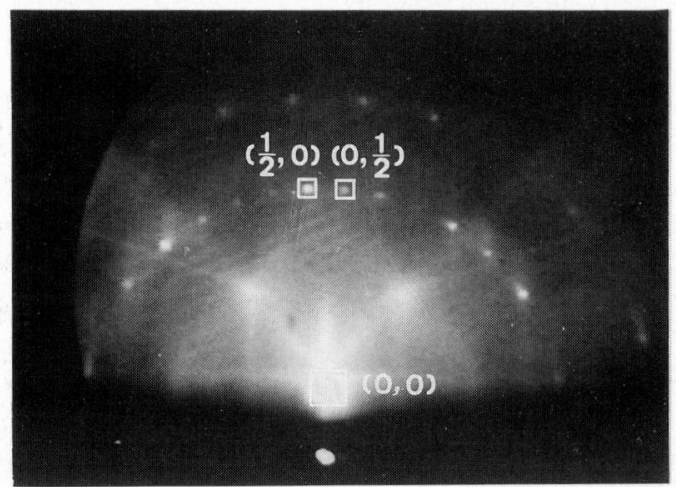

(c)

Fig. 4 RHEED patterns of (a) the Sb-saturated Si(001) surface at $\theta_{\mathrm{sb}}=\sim 1 \mathrm{ML}$, (b) a Sb-covered $\mathrm{Si}(001)$ surface at $\theta_{\mathrm{sb}}=\sim 0.7 \mathrm{ML}$ and (c) at $\theta_{\mathrm{sb}}=\sim 0.1 \mathrm{ML}$ during the desorption at $723{ }^{\circ} \mathrm{C}$. Corresponding Sb desorption times are marked on the top of Fig. 3.

intensity continues to increase gradually. Thus, the changes in the RHEED intensities occur just at $\theta_{\mathrm{Sb}} \sim 0.3$ $\mathrm{ML}$ where the time evolution of $\theta_{\mathrm{Sb}}$ shows a break, that is, an increase of Sb desorption rate. This means that the change in Sb desorption kinetics at $\theta_{\mathrm{Sb}} \sim 0.3 \mathrm{ML}$ is associated with the surface structural change of Sb layers. As $\theta_{\mathrm{Sb}}$ decreases further, the $(0,1 / 2)$ spot intensity increases again and saturates together with the $(1 / 2,0)$ spot intensity. The final saturation is due to the completion of $\mathrm{Sb}$ desorption as seen in $\theta_{\mathrm{sb}}$.

These observations are explained by considering the behavior of Sb-dimers on the major domains as in the case of $\mathrm{Bi}$ desorption on $\mathrm{Si}(001)^{15}$. The $\mathrm{Sb}$ atoms dimerise, forming the Sb-dimer rows perpendicular to the underlying Si-dimer rows as previously reported ${ }^{8,9)}$. Because $\mathrm{Sb}$ is larger in atomic size than $\mathrm{Si}$, the $\mathrm{Sb}$ layer has essentially a large strain. This strain may induce a variety of defects such as voids and anti-phase boundaries in the Sb layer which was observed by STM ${ }^{8,9)}$. The streaky RHEED patterns in Fig. 4 (a) and (b) are due to the anti-phase boundaries. Since the anti-phase boundary density decreases with decrease in $\theta_{\mathrm{Sb}}$ during Sb desorption, the $(-1,1)$ streak intensity could decrease. In the area where Sb desorbs, Si-dimers may appear, leading to the gradual increase in $(1 / 2,0)$ spot intensity as observed.

Then, the area of Sb layer with $(1 \times 2)$ periodicity on the major domains should decrease with decrease in $\theta_{\mathrm{sb}}$ causing decrease in $(0,1 / 2)$ spot intensity. However, the $(0,1 / 2)$ spot intensity increases as seen in Fig. 3. This is probably because that the $(1 \times 2)$ Sb layers are initially divided into small domains by anti-phase boundaries and that the coherency among domains increases with the decrease in $\theta_{\mathrm{sb}}$ as the anti-phase domain boundary diminishes. The temporary saturation in $(0,1 / 2)$ spot intensity at $\theta_{\mathrm{sb}} \sim 0.3 \mathrm{ML}$ suggests that the $(1 \times 2) \mathrm{Sb}$ domain size become too small and the contribution from the $(1 \times 2)$ minor domains of substrate become effective afterward. This is because the sharpness of $(0,1 / 2)$ spot recovers as seen in Fig.4 (c). It is important to note that the $(0,0)$ spot intensity at $\theta_{\mathrm{Sb}} \sim 0.3 \mathrm{ML}$ is already close to that for the clean surface. This means that Sb may not form domains but distributes randomly as Sb-dimers for $\theta_{\mathrm{Sb}}<\sim 0.3$ ML at high temperatures of $680-770^{\circ} \mathrm{C}$. This is consistent with the argument for the temporary saturation of the $(0,1 / 2)$ spot intensity.

Since the $\mathrm{Sb}$ desorption is found to be the first order in both the regions I and II, Sb desorption may occur locally from Sb-dimers. Therefore, it is understandable that the activation energies are almost the same in the regions I and II. However, it is found that the pre-exponential factors are different in the two regions. To understand this phenomenon, the argument about the spatial distribution of Sb-dimers as described above may be helpful. Namely, Sb-dimers are expected to form domains in region I but to be sparsely distributed in region $\mathrm{II}^{17,18)}$. If the $\mathrm{Sb}$ de- 
sorption is affected by the presence of nearest neighbor Sb-dimers, that would appear in desorption kinetics. This seems to have appeared in the pre-exponential factors. It may be worthwhile to note here that the desorption species are found to be $\mathrm{Sb}$-monomers ${ }^{\eta}$, whereas the units of $\mathrm{Sb}$ layers are $\mathrm{Sb}$-dimers. An atomistic kinetic model of $\mathrm{Sb}$ desorption is necessary to understand these phenomena.

In a previous study for a $\mathrm{Bi} / \mathrm{Si}(001)$ system, we reported that the Bi desorption obeys a first-order kinetics and the kinetics parameters hardly change in spite of the changes in long-range order of Bi layers; $(n \times 2)$ to $(1 \times$ $2)$ at $\sim 0.8 \mathrm{ML}$ and $(1 \times 2)$ to $(2 \times 2)$ at $\sim 0.4 \mathrm{ML}^{15}$. For all the $(n \times 2),(1 \times 2)$ and $(2 \times 2)$ structures, the local-binding unit is $\mathrm{Bi}$-dimer. The $\mathrm{Bi}$ desorption is expected to occur locally at Bi-dimer for all $\theta_{\mathrm{Bi}}$ as in the case of $\mathrm{Sb}$ desorption. However, the domain structure of $B i$ layers remains over a change in long-range order from $(1 \times 2)$ to $(2 \times 2)$ at $\theta_{\mathrm{Bi}} \sim 0.4 \mathrm{ML}$. Thus, the persistent domain morphology may well explain the single Bi desorption kinetics and make a sharp contrast to the case of $\mathrm{Sb}$.

\section{Summary}

The $\mathrm{Sb}$ coverage and surface order for the Sb desorption on a single domain $\mathrm{Si}(001) 2 \times 1$ surface were monitored in-situ and simultaneously using grazing-incidence RHEED-AES. It is found that the Sb desorption kinetics are divided into two Sb coverage regions; (I) $1 \mathrm{ML}>$ $\theta_{\mathrm{sb}}>\sim 0.3 \mathrm{ML}$ and (II) $\sim 0.3 \mathrm{ML}>\theta_{\mathrm{sb}}>\sim 0 \mathrm{ML}$. In both regions, $\mathrm{Sb}$ desorption is a first-order reaction and the activation energies of $\mathrm{Sb}$ desorption are almost the same; (I) $3.61 \mathrm{eV}$ and (II) $3.63 \mathrm{eV}$. However, the pre-exponential factors of the Sb desorption are different; (I) $2.9 \times 10^{15}$ $\mathrm{s}^{-1}$ and (II) $5.0 \times 10^{15} \mathrm{~s}^{-1}$. This is explained by a change in distribution of Sb-dimers as monitored by RHEED; a domain structure in (I) and a sparsely distributed one in (II).

\section{Acknowledgment}

The authors are grateful to Dr. K. Sakamoto and Dr. T. Sakamoto of Electrotechnical Laboratory for providing the well-oriented $\mathrm{Si}(001)$ wafers used in this study.

\section{References}

1) M.A. Boshart, A.A. Bailes III and L.E. Seiberling: Phys. Rev. Lett. 77, 1087 (1996).

2) M. Copel, M.C. Reuter, M.H. von Hoegen and R. M. Tromp: Phys. Rev. B 42, 11682 (1990).

3) K. Sakamoto, K. Miki, T. Sakamoto, H. Matsuhata and K. Kyoya: J. Cryst. Growth 127, 392 (1993).

4) S. Kono, T. Goto, Y. Okura and T. Abukawa: Jpn. J. Appl. Phys. 35, L1211 (1996).

5) K. Nakagawa, M. Miyao and Y. Shiraki: Jpn. J. Appl. Phys. 27, L2013 (1988).

6) J. Wasserfall and W. Ranke: Surf. Sci. 331, 1099 (1995).

7) S.A. Barnett, H.F. Winters and J.E. Greene: Surf. Sci. 165, 303 (1986).

8) M. Richter, J.C. Woicik, J. Nogami, P. Pianetta, K.E. Miyano, A.A. Baski, T. Kendelewicz, C.E. Bouldin, W.E. Spicer, C.F. Quate and I. Lindau: Phys. Rev. Lett. 65, 3417 (1990).

9) J. Nogami, A.A. Baski and C.F. Quate: Appl. Phys. Lett. 58, 475 (1991).

10) R.A. Metzger and F.G. Allen: Surf. Sci. 137, 397 (1984).

11) W.F.J. Slijkerman, P.M. Zagwijn and J.F. van der Veen: Surf. Sci. 262, 25 (1992).

12) A.V. Zotov, V.G. Lifshits and A.N. Demidchik: Surf. Sci. Lett. 274, L583 (1992).

13) A.V. Zotov, V.G. Lifshits and A.N. Demidchik: Sov. Tech. Phys. Lett. 18, 187 (1992).

14) K.-S. Kim, Y. Takakuwa, T. Abukawa and S. Kono: to be published in J. Cryst. Growth.

15) K.-S. Kim, Y. Takakuwa, Y. Mori and S. Kono: Jpn. J. Appl. Phys. 35, L1695 (1996).

16) Y. Enta, S. Suzuki, S. Kono and T. Sakamoto: Phys. Rev. B 39, 5524 (1989).

17) Y.W. Mo: Phys. Rev. Lett. 69, 3643 (1992).

18) Y.W. Mo: J. Vac. Sci. Technol. B 12, 762 (1994). 